\section{Regards sur l'économie allemande}

Bulletin économique du CIRAC

$70 \mid 2005$

Varia

\title{
Chine, Inde, Brésil : les choix des entreprises allemandes
}

Markus Gabel

\section{OpenEdition}

1 Journals

Édition électronique

URL : http://journals.openedition.org/rea/2093

DOI : 10.4000/rea.2093

ISBN : 978-2-8218-0836-2

ISSN : 1965-0787

\section{Éditeur}

CIRAC

Édition imprimée

Date de publication : 1 mars 2005

Pagination : 31-34

ISSN : 1156-8992

\section{Référence électronique}

Markus Gabel, «Chine, Inde, Brésil : les choix des entreprises allemandes », Regards sur l'économie allemande [En ligne], 70 | mars 2005, mis en ligne le 16 décembre 2008, consulté le 14 novembre 2019. URL : http://journals.openedition.org/rea/2093 ; DOI : 10.4000/rea.2093 


\title{
Chine, Inde, Brésil : les choix des entreprises allemandes
}

\author{
Markus Gabel
}

Plus personne ne conteste l'ascension de la Chine, de l'Inde et du Brésil parmi les puissances économiques. Pourtant, l'Allemagne, championne mondiale à l'export, joue pour l'instant essentiellement la carte chinoise. Les chiffres sont parlants : tandis que les échanges germano-chinois se sont élevés à près de 44 milliards $€$ en 2003, ceux avec le Brésil n'ont pas atteint 8 milliards; avec I'Inde, ils ont tout juste dépassé les 5 milliards. Ces chiffres traduisent une approche très différente de ces trois pays : la Chine est un marché prioritaire pour les grandes entreprises et le devient pour le Mittelstand, car contrairement aux deux autres pays, elle est perçue à la fois comme un site de production et, du fait de la taille comme du pouvoir d'achat de son marché intérieur, comme un débouché incontournable pour le made in Germany. La liste des firmes présentes se lit ainsi comme le who's who de l'économie allemande. Et la Chine est aujourd'hui le premier partenaire économique allemand en Asie - devant le Japon. Depuis son arrivée au pouvoir en 1998, le chancelier Schröder a déjà visité la Chine à six reprises.

Les relations économiques sino-allemandes ont enregistré une évolution extraordinaire. En trente ans, le volume des échanges commerciaux s'est multiplié par 100, passant de 0,445 milliard $€$ en 1972 à 43,9 milliards $€$ en 2003 , ce qui représente près de $40 \%$ du volume des échanges franco-allemands (177 milliards $€$ en 2003). Au fil des ans, l'excédent commercial initial face à la Chine s'est transformé en déficit. II reste néanmoins plutôt faible et oscille depuis des années entre 5 et 9 milliards $€(7,4$ milliards $€$ en 2003). Convoitée depuis longtemps, la Chine est depuis 2002 le second marché à l'export hors l'Europe après les Etats-Unis. Si on inclut les exportations vers la zone de Hong Kong, l'espace économique chinois l'est depuis 2000 déjà.

Les échanges sont relativement plus importants du côté chinois : pour ce dernier, l'Allemagne est le sixième partenaire commercial. Pour l'Allemagne, la Chine ne figure qu'à la $10^{\mathrm{e}}$ place en termes d'exportations, avec $2,7 \%$ du total en 2003. Et ceci malgré la croissance exceptionnelle des exportations allemandes ces dernières années (entre $20 \%$ et $30 \%$ depuis 2001). Les importations ont enregistré une croissance un peu moins soutenue (entre $7 \%$ et $17 \%$ depuis 2001), réduisant ainsi le défit commercial allemand avec la Chine - une tendance exceptionnelle parmi les grands pays industrialisés.

Les exportations allemandes vers la Chine sont très concentrées : les secteurs de l'automobile et de la machine-outil en fournissent près de $55 \%$. Le troisième secteur, certes distancé par les deux précédents, est celui de la production et distribution d'électricité (8,5\% en 2003). Les firmes allemandes, très en pointe dans la production d'énergies propres (voir REA 57/02), profitent ici d'une préoccupation de plus en plus marquée des autorités chinoises pour l'écologie. La part de ces trois secteurs s'est nettement accentuée depuis 2000, passant de $45 \%$ à $63 \%$ en 2003. Cette progression s'est réalisée au détriment relatif des télécommunications, de la bureautique et de l'électronique grand public. La concentration est nettement moins prononcée du côté des importations qui reflètent encore le caractère de PED de la Chine, qui reste un marché à très bas salaires

Les échanges sino-allemands multiplié par 100 depuis 1972

Déficit commercial en recul

Forte concentration des exportations vers la Chine 
avec un PIB par tête de 1000 \$ en 2003. Trois secteurs couvrent chacun $16 \%$ à $17 \%$ des importations : textile-habillement-cuir, bureautique-informatique et électroménager-télécommunication. Mais on observe une tendance à la spécialisation : le poids des trois secteurs est passé de $44 \%$ à $50 \%$ depuis 2000.

Les échanges de I'Allemagne avec la Chine et I'Inde (valeur en milliards d'euros)

\begin{tabular}{|l|cccc|cccc|}
\hline & \multicolumn{4}{|c}{ Chine } & \multicolumn{4}{c|}{ Inde } \\
& $\mathbf{2 0 0 0}$ & $\mathbf{2 0 0 1}$ & $\mathbf{2 0 0 2}$ & $\mathbf{2 0 0 3}$ & $\mathbf{2 0 0 0}$ & $\mathbf{2 0 0 1}$ & $\mathbf{2 0 0 2}$ & $\mathbf{2 0 0 3}$ \\
Exportations allemandes & 9,46 & 12,12 & 14,57 & 18,26 & 2,08 & 2,31 & 2,42 & 2,44 \\
Importations allemandes & 18,55 & 19,94 & 21,34 & 25,68 & 2,46 & 2,52 & 2,54 & 2,64 \\
Solde commercial & $-9,09$ & $-7,82$ & $-\mathbf{6 , 7 7}$ & $-\mathbf{- 7 , 4 2}$ & $-\mathbf{- 0 , 3 8}$ & $-\mathbf{0 , 2 1}$ & $\mathbf{- 0 , 1 2}$ & $-\mathbf{0 , 2 0}$ \\
\hline
\end{tabular}

Source des données : Destatis, janvier 2005 (www.destatis.de).

L'Allemagne est le plus grand investisseur européen
Ces évolutions commerciales reflètent une intense activité en termes d'investissements. A la fin 2003, le stock d'investissement des entreprises allemandes en Chine s'élève à 9,3 milliards $€$; c'est largement 10 fois plus qu'en 1995 (800 millions). Les investissements proviennent surtout de quatre secteurs: l'automobile avec $30 \%$ (VW, BMW et prochainement DaimlerChrysler), l'industrie électronique avec $15 \%$, la chimie avec près de $10 \%$ (BASF et Bayer notamment) et la machine-outil (près de $6 \%$, surtout des PME). Depuis 1999, l'Allemagne est le plus grand investisseur européen en Chine devant le Royaume-Uni. Mais comparativement, le volume reste modeste. L'Allemagne n'arrive ainsi qu'à la neuvième place des investisseurs, loin derrière Hong Kong, les Etats-Unis, Taiwan et la Corée.

Les relations avec le Brésil, délaissées en comparaison
Le traditionnel excédent commercial avec le Brésil s'est récemment réduit $(0,24$ milliard $€$ en 2003 après
1,12 milliard en 2002), suite à une forte baisse des exportations allemandes (4,1 milliards $€$ en 2003
après 4,9 en 2002) contractant le volume des échanges à 7,9 milliards $€$ en 2003 . Commercialement, le
Brésil est très tourné vers l'Allemagne qui couvre $10 \%$ de son commerce extérieur (ce qui vaut à la RFA
la troisième place en termes d'échanges après les USA et l'Argentine). Cette importance ne se reflète pas
dans les investissements où l'Allemagne ne prend plus que la neuvième place (0,5 milliard $€$ en 2003$)$.
Le plus grand pays d'Amérique latine, et tête de pont de l'industrie allemande vers le marché nord-améri-
cain depuis... la fin du XIX siècle, accuse une nette baisse d'intérêt depuis que l'Europe de l'Est est de-
venue la destination prioritaire pour la relocalisation d'activités industrielles (voir REA $59 / 02)$. Malgré tout,
le stock d'investissement allemand au Brésil s'élève encore à 14 milliards $€-$ grâce surtout aux années
1970/80 durant lesquelles trois quarts des investissements allemands vers les PVD allaient vers l'Amé-
rique du Sud, dont $70 \%$ au Brésil. Mais les temps ont changé : en 1979, le stock d'investissement alle-
mand au Brésil était 10 fois plus important que celui en Asie ; aujourd'hui, il ne l'est plus que de trois fois.
Néanmoins, l'héritage de ces investissements fait que les liens restent solides et même exceptionnels : le
Brésil abrite ainsi la plus grande ville industrielle 'allemande', Sao Paulo. On y trouve la plupart des 1200
filiales d'entreprises allemandes, conséquence non seulement du poids économique de la ville et de sa
région, mais aussi de sa proximité avec les régions de prédilection de l'importante immigration allemande
au Brésil, surtout concentrée au Sud du pays depuis le début de l'ère industrielle.

Chine : un marché difficile pour le Mittelstand

La promotion des échanges est assurée par de nombreux acteurs
Selon un sondage effectué par la Deutsche Bank au début 2004 parmi les plus grandes entreprises, les investissements allemands en Chine pourraient monter à 20 milliards $€$ en 2010. La conséquence pourrait être une diversification de la localisation des investissements allemands, concentrés pour l'instant dans trois régions : Shanghai et le delta du Yangzi Jiang (qui comptent les plus anciennes implantations allemandes), le nord-est chinois (très industriel et proche de Pékin) et la région du Guangdong (capitale : Canton), dominée par les PME. Mais malgré l'indéniable attractivité du marché chinois, les entreprises, surtout les PME, restent insatisfaites de la fiabilité comme de la transparence juridiques et se plaignent d'une discrimination lors des appels d'offres. Le plus grand problème reste cependant le vol d'idées et l'insuffisance de la protection des brevets. L'Allemagne a ainsi insisté pour que soit signé en 2003 un traité remédiant à cette situation (Investitionsschutz- und fördervertrag).

L'Allemagne dispose d'un arsenal bien équipé pour promouvoir ses intérêts en Chine. Elle entretient plusieurs bureaux de délégués (à Pékin, Hong Kong, 
Shanghai et Canton) organisés sous le toit du département des relations extérieures (Auslandskammerwesen) de la Chambre allemande d'industrie et du commerce. L'agence pour la promotion du commerce extérieur (Bundesagentur für Außenwirtschaftsförderung, BfAl ; www.bfai.org) a sur place des correspondants à qui elle fournit une aide logistique. Ces acteurs sont organisés dans un réseau qui travaille en lien étroit avec l'ambassade et les différents consulats. Par ailleurs, à Pékin existe le German Center Beijing (il appartient à la Landesbank Baden-Württemberg), qui soutient surtout les PME dans leur recherche de locaux. II faut citer également le Ostasiatischer Verein et l'Asien-Pazik-Ausschuss der deutschen Wirtschaft, organismes privés qui agissent 'à distance', c'est-à-dire à partir d'Allemagne, en faveur des intérêts allemands dans la région, ainsi que l'inévitable Euler-Hermes-Kredit AG, institution entièrement publique qui distribue des crédits à l'exportation.

Les relations avec l'Inde se distinguent à plus d'un titre de celles avec la Chine. D'abord, par leur moindre volume : en 2003, elles ont atteint à peine plus de $11 \%$ du commerce germano-chinois. Ensuite, on est également loin du dynamisme extraordinaire des relations avec la Chine, car les échanges commerciaux avec l'Inde marquent le pas: depuis 2000 , le volume stagne entre 4,5 et 5 milliards $€$. Enfin, l'importance relative des échanges est encore plus déséquilibrée que dans le cas de la Chine: pour l'Inde, l'Allemagne arrive en $5^{\mathrm{e}}$ position ; l'Inde n'apparaît pas parmi les 30 principaux partenaires de l'Allemagne.
Statu quo avec I'Inde à faible niveau

La structure des échanges allemands avec la Chine et l'Inde en 2003 (en \%)*

\begin{tabular}{|l|cc|rc|}
\hline & $\begin{array}{c}\text { Imp. chinoises } \\
\text { en RFA }\end{array}$ & $\begin{array}{c}\text { Exp. all. vers } \\
\text { la Chine }\end{array}$ & $\begin{array}{c}\text { Imp. indiennes } \\
\text { en RFA }\end{array}$ & $\begin{array}{c}\text { Exp. all. vers } \\
\text { I'Inde }\end{array}$ \\
Articles textiles & 2,91 & 0,47 & 14,04 & 1,07 \\
Vêtements & 9,90 & 0,08 & 20,38 & 0,06 \\
Cuir et articles de maroquinerie & 4,04 & 0,04 & 8,81 & 0,28 \\
Produits chimiques & 3,81 & 6,69 & 15,84 & 17,44 \\
Métaux et produits métallurgiques / sidérurgiques & 0,77 & 5,64 & 1,86 & 8,92 \\
Métallurgie & 3,91 & 2,45 & 2,94 & 2,23 \\
Machine outil & 6,20 & 30,90 & 5,08 & 30,41 \\
Bureautique et équipement informatique & 16,64 & 0,75 & 0,41 & 1,29 \\
Production et distribution d'électricité & 9,68 & 8,49 & 3,48 & 7,28 \\
Télécommunications, électronique grand public & 16,99 & 4,01 & 2,57 & 3,25 \\
Appareils médicaux et de mesure, optique, montres & 3,17 & 5,13 & 1,34 & 9,37 \\
Voitures et pièces détachées & 0,43 & 19,58 & 2,51 & 2,69 \\
Utilitaires et autres automobiles & 1,38 & 3,92 & 1,30 & 4,01 \\
Meubles, bijoux, instruments de musique et de sport, jeu & 11,42 & 0,95 & 4,10 & 0,65 \\
Divers (dont : produits agricoles, verre, céramique ...) & 8,75 & 10,9 & 15,34 & 11,05 \\
\hline
\end{tabular}

Source des données: Destatis, janvier 2005 (www.destatis.de). *) En pourcentage du total des importations chinoises / indiennes vers l'Allemagne, respectivement exportations allemandes vers la Chine/l'Inde.

Si le marché indien reste peu important pour l'Allemagne (0,5\% des échanges), les secteurs présents sont les mêmes que chez le voisin chinois. La construction mécanique et les produits chimiques représentent près de $60 \%$ des ventes en Inde. Des secteurs jusqu'ici secondaires ont récemment enregistré des taux de croissance importants, comme l'électronique et la métallurgie. Du côté des importations indiennes vers l'Allemagne, la concentration est encore plus marquée : plus de $43 \%$ proviennent du secteur textile-vêtement-cuir. Mais il est très incertain que ce secteur puisse maintenir cette position, compte tenu de la future percée chinoise à l'échéance de l'accord multi-fibres le $1^{\mathrm{er}}$ janvier 2005, qui libéralise complètement ce secteur. L'importance relative du secteur a déjà reculé (de $50 \%$ en 2000). Etant donné la légère progression des importations globales, cette baisse traduit une diversification des ventes indiennes vers
Les poids lourds allemands toujours présents en Inde 
Forte baisse des investissements allemands en Inde

Un important potentiel inexploité
l'Allemagne. Elle concerne les produits chimiques (16\% en 2003 contre $10 \%$ en 2000), mais également l'électrotechnique, l'automobile, les appareils médicaux et l'optique.

Bien que depuis 1991, c'est-à-dire le début de la politique de réformes en Inde, environ 2560 joint ventures aient vu le jour (144 en 2003), le montant des investissements annuels est en baisse, tombant de 480 millions $€$ en 1997 à 51,7 millions $€$ en 2002 et à 37 millions $€$ en 2003. Les investissements réalisés concernent surtout la chimie, la pharmacie, la machine-outil et l'électronique, et ils s'élèvent en 2003 à un stock de 1,7 milliard €. La position allemande en Inde reste faible : bien que troisième investisseur européen (après le Royaume-Uni et les Pays-Bas), l'Allemagne n'assure que $5 \%$ des IDE indiens.

Rares sont pour l'instant les entreprises allemandes à avoir osé s'implanter en Inde, surtout parmi les PME. Le producteur de chaussures Bär (Bietigheim, Bade-Wurtemberg) est une des rares exceptions. Quant aux grandes entreprises, lorsqu'elles sont présentes, elles le sont encore trop souvent dans des niches, ainsi DaimlerChrysler, Porsche et autres sur le marché des voitures de luxe. Mais une amélioration pourrait venir des secteurs qui font actuellement le succès de l'Inde : la délocalisation d'activités de call-centers, de comptabilité et de R\&D - des domaines dans lesquels l'Allemagne est presque absente jusqu'ici. Conscients de ce potentiel, les gouvernements ont signé fin 2003 l'Agenda For Idian-German Partnership for the Twenty-first-century. Celui-ci donnera un nouveau souffle à l'institution centrale des relations germano-indiennes, la Deutsch-Indische Handelskammer qui est, avec ses 6500 membres, la plus grande des chambres de commerce bilatérales. Un des axes centraux du développement est l'environnement: les entreprises allemandes sont en train de réaliser une percée sur le marché des énergies renouvelables en Inde, surtout grâce à leur expertise dans le domaine des éoliennes.

POUR L'INSTANT, LE COMMERCE extérieur allemand avec les trois futurs géants ne connaît qu'une destination : la Chine. Ce pays est surtout perçu comme le plus grand marché du monde, tandis que l'Inde souffre encore de son image de PVD. Le Brésil, quant à lui, a été doublé par l'Europe de l'Est en termes de lieux de production bon marché. Mais cette solution de proximité pourra rapidement toucher à ses limites si le processus de rattrapage des nouveaux membres de l'UE se poursuit à la même vitesse. Or l'Inde et le Brésil ont un atout encore largement inexploité par les firmes allemandes : la localisation de services. L'Allemagne a pour l'instant peu d'expérience dans ce domaine, mais le potentiel est identifié : en Asie, avec son réservoir énorme de main d'œuvre très qualifiée, l'Inde complèterait ainsi parfaitement la Chine, considérée surtout comme « établi prolongé ». D'autres pays, surtout anglo-saxons, l'on déjà compris, améliorant de la sorte leur compétitivité. Le Brésil peut jouer le même rôle pour l'Amérique du Sud. Avec son système de décision économique, politique et juridique plus proche de la culture européenne, il dispose d'un avantage de poids par rapport à l'Inde. Mais en dépit des relations diplomatiques et de l'intérêt affiché du chancelier pour la Chine, les choix mondiaux de localisation des activités relèvent outre-Rhin des seules entreprises. Et leurs critères premiers sont la compétitivité de ces géants, de même que leur importance stratégique pour la spécialisation mondiale des entreprises allemandes.

\section{Indications bibliographiques}

GABEL M., « Commerce extérieur allemand : faiblesses et atouts », Regards sur l'économie allemande, $\mathrm{n}^{\circ} 63 / 03$

GRZISKA M., « Chinas Integration in die Weltwirtschfat - Chancen und Risiken », DIW Wochenbericht, $\mathrm{n}^{\circ} 52,2004$

TRINH T., «Deutsche Investitionen in China - Chance für deutsche Unternehmen? », Deutsche Bank Research, 6 août 2004 